\title{
Case Report of a Benign Looking Malignant ECG- Value of Subtle Signs
}

\author{
Khushal Lokchand Sharnagat ${ }^{1}$ Deepthi Kodati ${ }^{1}$ \\ ${ }^{1}$ Department of Cardiology, Care Hospital, Nampally, Hyderabad, \\ Telangana, India
}

Ind J Car Dis Wom 2021;6:35-38.

\begin{abstract}
Address for correspondence Khushal Lokchand Sharnagat, MD, Department of Cardiology, Care Hospital, Nampally, Hyderabad, Telangana-500001, India (e-mail: klsharnagat@gmail.com).
\end{abstract}

\begin{abstract}
Keywords

- electrocardiography

- cardiac arrest

- left main disease

ECG is one of the primary and most informative bedside tools for the cardiologist, even in the present era. Careful analysis of ECG changes (sometimes subtle) can be life-saving, such as in the case we are presenting now. A 42-year-old gentleman with no obvious coronary risk factors presented with vague pain and subtle ECG changes (outside physician passed off as usual). He was asked to consult a cardiologist. While awaiting outpatient consultation, he collapsed. The patient was resuscitated and coronary angiogram was immediately done, which showed distal left main disease. Successful intervention was carried out with the help of provisional stent strategy, and the patient was discharged safely.
\end{abstract}

\section{Introduction}

Electrocardiography is the primary and earliest performed cardiac investigation for any patient. However, it can be confusing and misleading in many cases. It is also one of the most neglected tests in the present era (especially with the availability of echo and other advanced techniques). Therefore, we report the case of a 42-year-old male who presented with chest pain and minor changes in ECG. The patient was taken for angiogram, and angioplasty was done immediately, which was life-saving, and the patient was successfully discharged. The purpose of presenting this case is to highlight the careful assessment of the ECG and how it can be life-saving, as shown in this case.

\section{Case Details}

A 42-year-old gentleman, who was nonhypertensive, nondiabetic, and a non-smoker, presented with complaints of vague abdominal and lower chest discomfort for 6 hours. He initially visited a physician close to his house. ECG was taken and reported as usual ( - Fig. 1). The patient was subsequently sent home with antacids prescribed. In view of

published online April 9, 2021
DOI https://doi.org/ 10.1055/s-0041-1723922. ongoing discomfort, he visited our hospital. While he was waiting in the cardiology outpatient department, he collapsed (monitor suggestive of monomorphic ventricular tachycardia [VT]), following which cardiopulmonary resuscitation (CPR) was given. The patient revived after a couple of minutes, ECG was repeated, which was more or less the same as outside ECG. Bedside echocardiography showed hypokinetic mid and apical interventricular septum (IVS) and anterior wall. Subsequently, the patient was taken for an angiogram, which showed distal left main coronary artery (LMCA) disease and ostial left anterior descending (LAD) artery disease. Provisional stenting of LMCA to LAD was done with 4-22 Resolute Onyx (Medtronic) stent with the right final result. The patient was extubated on the next day and discharged 2 days later. At the time of discharge, an echocardiogram showed mild hypokinesia of apex and overall proper left ventricular function.

\section{Discussion}

ECG is a simple and vital tool available to cardiologists at the bedside. However, with the availability of several investigational modalities like echo and other imaging techniques, 


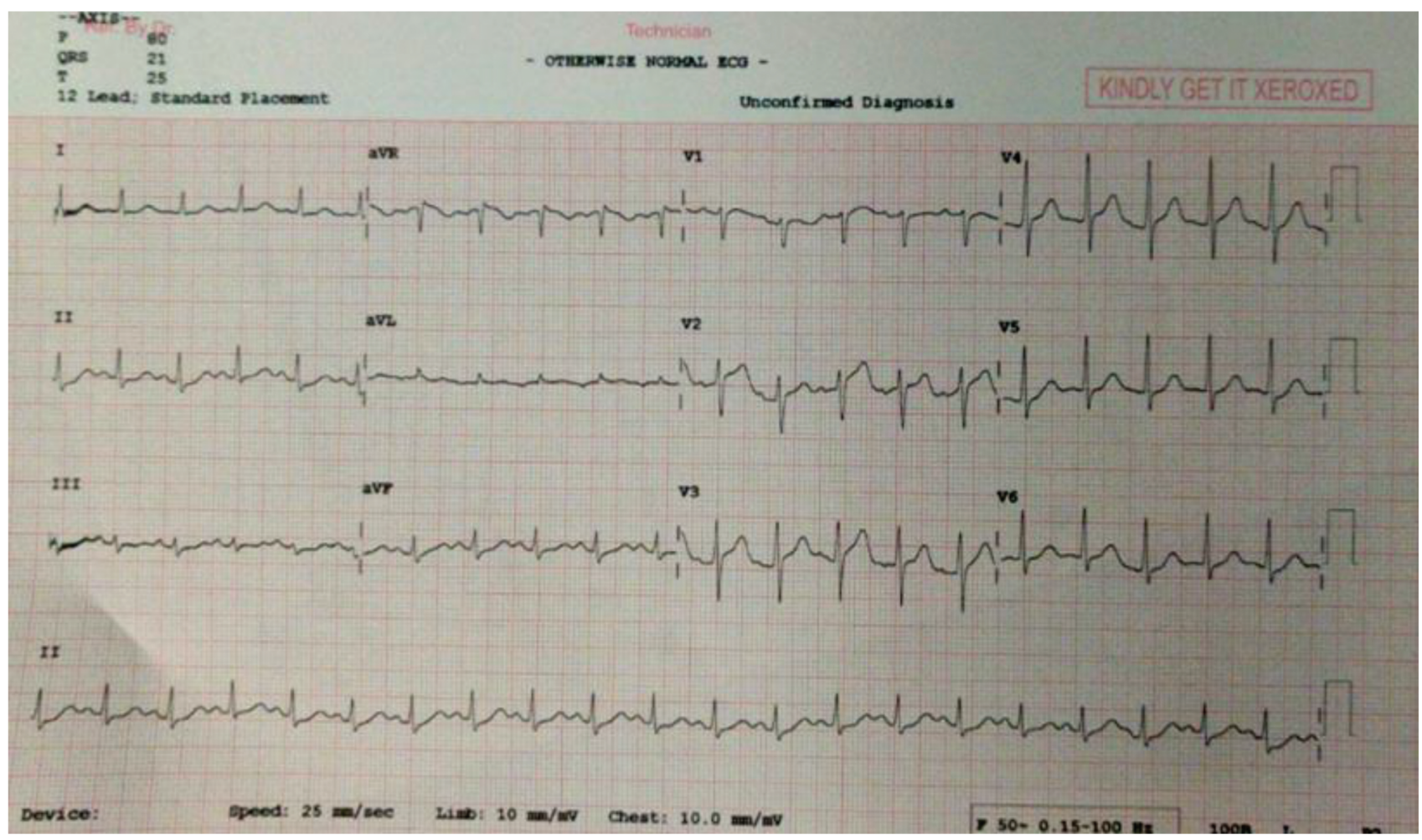

Fig. 1 Initial ECG.

it is noted that proper analysis of ECG is often ignored. It is observed that clinicians and even cardiologists not uncommonly spend very less time in evaluating ECGs. Hence, there is a probability of missing crucial signs which can be dangerous in the management of such cases (as in this case). This gentleman was attended to by an outside physician and ECG done but passed off as normal. However, on careful analysis, even initial ECG ( - Fig. 2) was showing the following important although subtle changes.

- Slight ST segment coving elevation in aVR.

- Minimal ST-segment depressions in lead 2 and aVF.

- Taller T waves in precordial leads (although T wave height not $>5 \mathrm{~mm}$, T waves are more than $50 \%$ of $\mathrm{R}$ wave amplitude in some right precordial leads).

It is well-known that aVR ST elevation and ST depression in other leads suggest LMCA disease (- Table $\mathbf{1}$ ).

Lead aVR is often described as "neglected lead," ${ }^{1}$ because it points away from the direction of left ventricular depolarization. Prominent changes also most of the time are ignored in this lead. The mechanism behind ST elevation in aVR is either transmural basal septal ischemia or transmural ischemia of the right ventricle. Noticeably, however, these changes are less pronounced in our case. Hence, it could have been missed. However, in a patient with chest and abdominal discomfort, the ECG findings, however subtle, ${ }^{2}$ should be given more weightage (-Table 2 ).

Two-dimensional (2D) echocardiography showed hypokinesia of apex and mid and apical IVS and anterior wall. This echo correlation further increases the importance of the accompanying ECG changes. Subsequently, angiogram showed distal LMCA disease ( - Fig. 3). It was thus confirming our judgment after viewing ECG.

We subsequently did a percutaneous coronary intervention (PCI) of LMCA to LAD with 4-22 Resolute Onyx stent with final good result (provisional stenting strategy) (-Fig. 4). The patient improved clinically. Postprocedure ECG showed resolution of the subtle changes noticed earlier ( - Fig. 5) and no further arrhythmic events noted postprocedure.

As evident in the postprocedure, ECG shows resolved ST elevation in aVR and no ST depressions in inferior leads.

Thus, taking this patient for early angiogram and revascularization was very useful. The presence of ongoing pain and the episode of cardiac arrest needing CPR all warranted early invasive strategy. ${ }^{3}$ Conservative strategy would be hazardous in a patient with this location of disease (distal LMCA and ostial LAD). In the setting of an acute coronary syndrome (ACS), ST elevation in aVR has been demonstrated to be the single most potent ECG predictor of significant LMCA stenosis, clearly outperforming other parameters such as thrombolysis in myocardial infarction (TIMI) score, troponin I, and brain natriuretic peptide levels. From a therapeutic standpoint, patients with such ECG findings benefit from early intervention. Early recognition of this pattern of clinical signs and ECG findings (although minor), could be critical for making the correct diagnosis and risk stratifying the patient for early coronary angiography and urgent revascularization. On the other hand, had the patient continued with antacids at home, the results could have been catastrophic. In our case, the patient was extubated the next day and discharged home a couple of days later. 


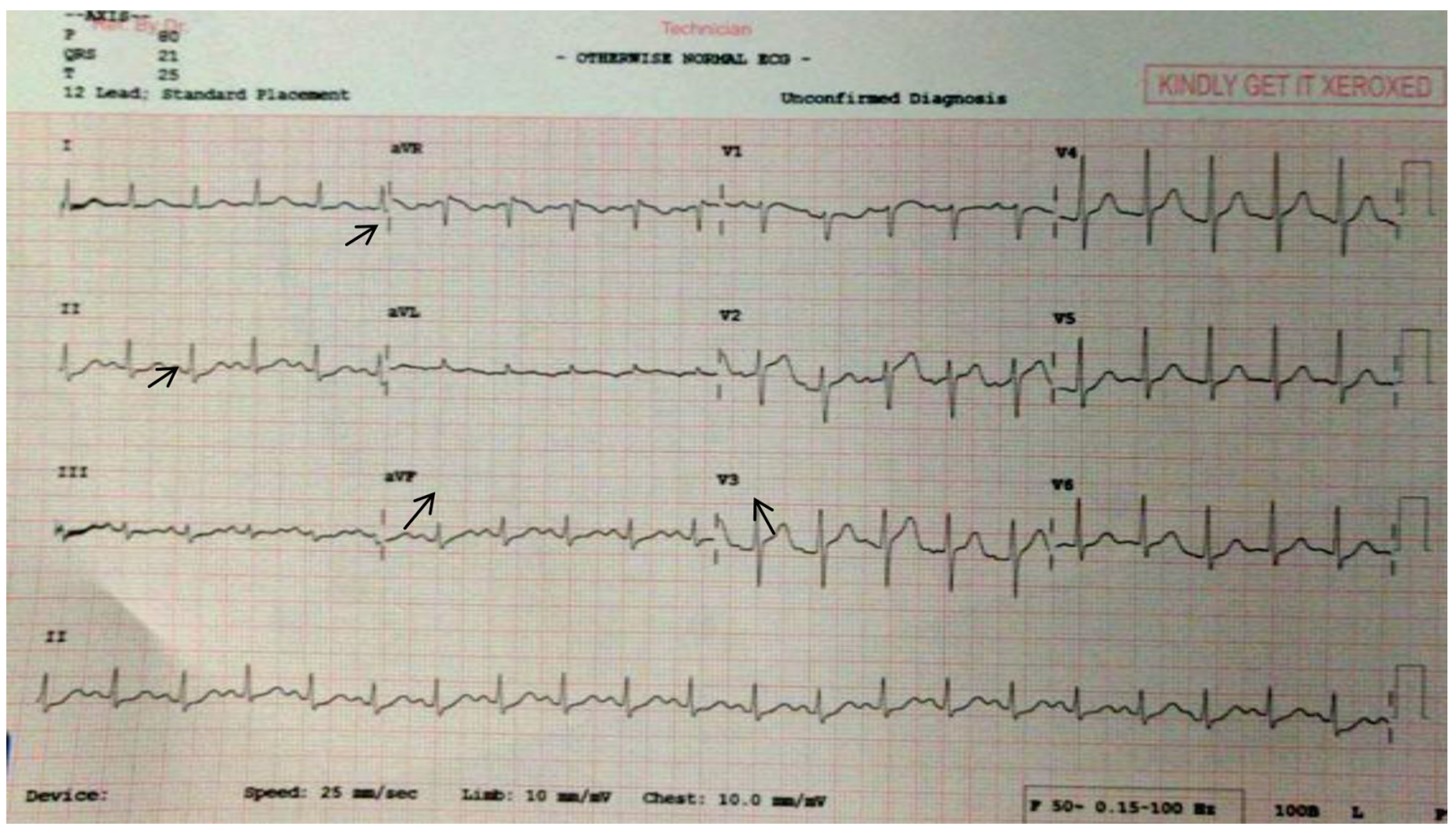

Fig. 2 Detailed analysis of initial ECG, showing subtle but important changes.

Table 1 Common ECG findings in LMCA disease ${ }^{4,5}$

\begin{tabular}{|l|}
\hline $\begin{array}{l}\text { 1. Widespread horizontal ST depressions, especially in the } \\
\text { lead } 1,2 \text {, and } \mathrm{V} 4-6 \text {. }\end{array}$ \\
\hline 2. ST elevation in $\mathrm{aVR}>$ or $=1 \mathrm{~mm}$ \\
\hline 3. ST elevation in $\mathrm{aVR}>$ or $=\mathrm{V} 1$ \\
\hline
\end{tabular}

Abbreviation: LMCA, left main coronary artery.

Table 2 Subtle ECG findings of critical ischemic heart disease

\begin{tabular}{|l|}
\hline $\begin{array}{l}\text { 1. Horizontal ST segment with ST making an acute angle } \\
\text { with T wave. }\end{array}$ \\
\hline 2. U wave inversion in the presence of upright T waves. ${ }^{6}$ \\
\hline 3. Tall peaked T waves. \\
\hline 4. Symmetric T inversions generalized. \\
\hline $\begin{array}{l}\text { 5. Isolated T inversion in aVL-may be first change seen in } \\
\text { acute inferior MI. }\end{array}$
\end{tabular}

Abbreviation: MI, myocardial infarction.

\section{Learning Points From this Case}

- Careful analysis of ECG is essential-changes in a group of leads (even if subtle) are more significant than severe changes in a single lead alone.

- Patient symptoms have to be given significance, even though ECG appears benign. Keeping such patients in observation units for serial ECGs and cardiac enzymes, if necessary, is useful.

- Careful observation of his initial ECG reflects the possibility of underlying LMCA disease. It could have quickened the initiation of therapy and probably have prevented the cardiac arrest episode if intervention had happened early.

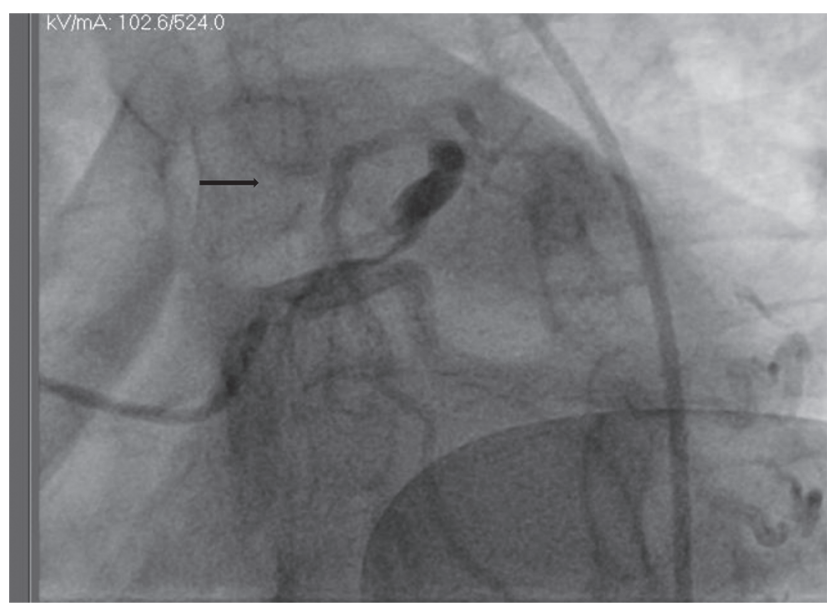

Fig. 3 Initial angiographic picture showing distal left main coronary artery (LMCA) and ostial left anterior descending (LAD) artery critical disease.

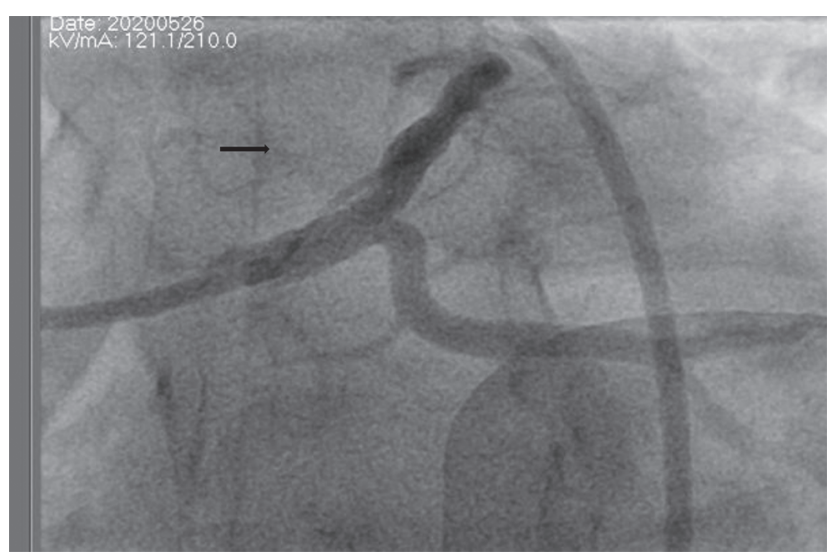

Fig. 4 Final angiographic result after stenting. 


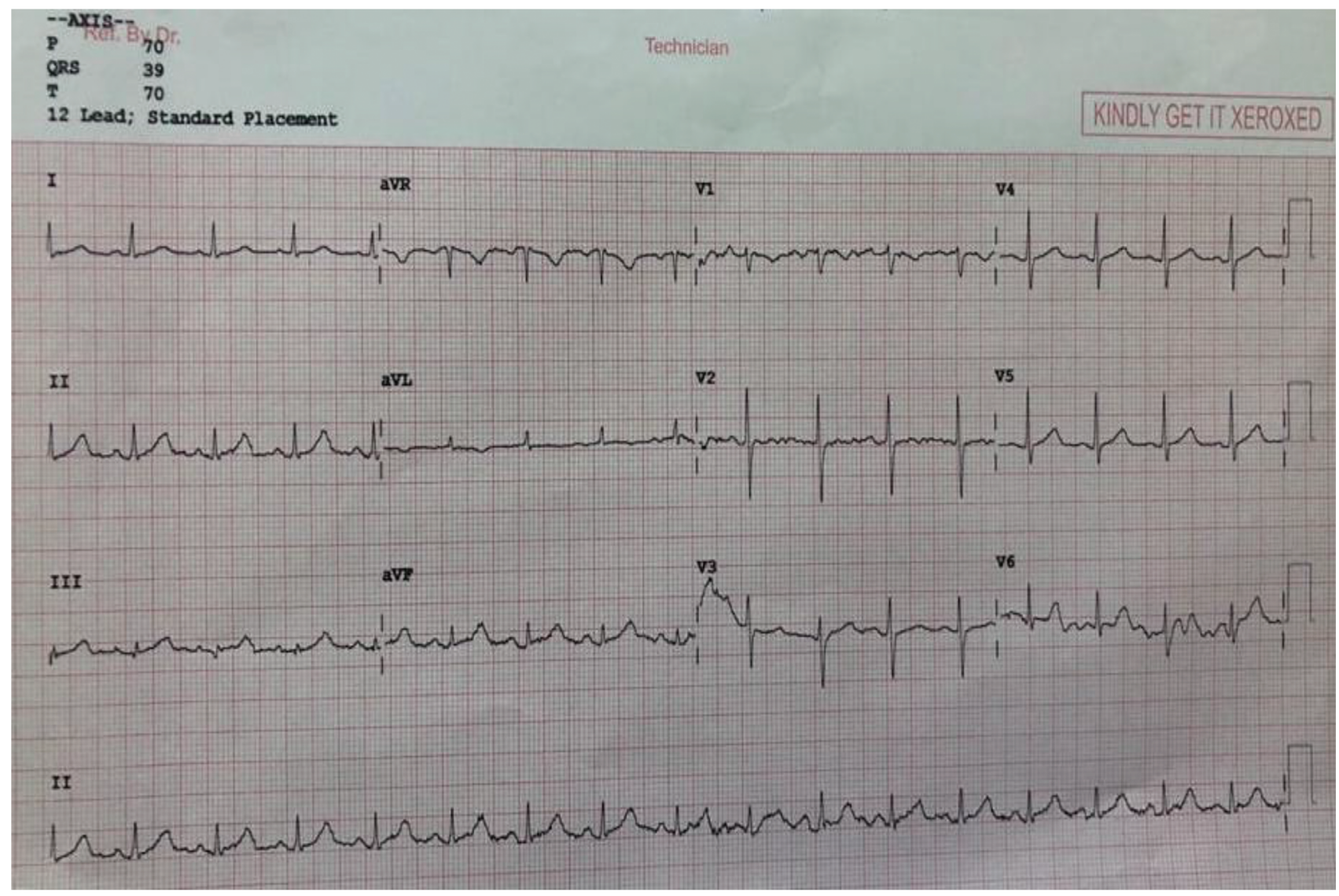

Fig. 5 Postprocedure ECG.

- Correlation with echocardiogram is beneficial, especially in patients with minimal changes in ECG.

\section{Conclusions}

Angina can sometimes be vague and have a multitude of presentations. ECG is a simple tool to assess the presence of coronary artery disease (CAD) and check for any high-risk features. It is imperative to check for even subtle changes and monitor ECGs serially, if necessary. Such careful analysis can prevent significant mistakes in the management of some patients and help us properly plan treatment options.

\section{Conflicts of Interest}

None declared.

\section{References}

1 Kireyev D, Arkhipov MV, Zador ST, Paris JA, Boden WE. Clinical utility of aVR-The neglected electrocardiographic lead. Ann Noninvasive Electrocardiol 2010;15(2):175-180
2 Tewelde SZ, Mattu A, Brady WJ Jr. Pitfalls in electrocardiographic diagnosis of acute coronary syndrome in low-risk chest pain. West J Emerg Med 2017;18(4):601-606

3 Puymirat E, Taldir G, Aissaoui N, et al. Use of invasive strategy in non-ST-segment elevation myocardial infarction is a major determinant of improved long-term survival: FAST-MI (French Registry of Acute Coronary Syndrome). JACC Cardiovasc Interv 2012;5(9):893-902

4 Sen F, Ozeke O, Kirbas O, et al. Classical electrocardiographic clues for left main coronary artery disease. Indian Heart J 2016;68(Suppl 2) :S226-S227

5 Chua K, Singh G, Yap J, et al. Electrocardiographic characteristics of left main coronary artery st-elevation myocardial infarction: results from a multicenter registry. J Am Coll Cardiol 2014;63(12, Supplement):A148

6 Kishida H, Cole JS, Surawicz B. Negative U wave: a highly specific but poorly understood sign of heart disease. Am J Cardiol 1982;49(8):2030-2036 